$1-1-1986$

\title{
Energy systematics of single and double K-shell-vacancy production in titanium bombarded by chlorine ions
}

James Hall

Patrick Richard

Philip L. Pepmiller

D. C. Gregory

P. D. Miller

See next page for additional authors

Follow this and additional works at: https://bearworks.missouristate.edu/articles-cnas

\section{Recommended Citation}

Hall, James, Patrick Richard, Philip L. Pepmiller, D. C. Gregory, P. D. Miller, C. D. Moak, C. M. Jones, and GD Alton, L. B. Bridwell, and C. J. Sofield. "Energy systematics of single and double K-shell-vacancy production in titanium bombarded by chlorine ions." Physical Review A 33, no. 2 (1986): 914.

This article or document was made available through BearWorks, the institutional repository of Missouri State University. The work contained in it may be protected by copyright and require permission of the copyright holder for reuse or redistribution.

For more information, please contact BearWorks@library.missouristate.edu. 


\section{Authors}

James Hall, Patrick Richard, Philip L. Pepmiller, D. C. Gregory, P. D. Miller, C. D. Moak, C. M. Jones, G. D. Alton, Lynn Boyd Bridwell, and C. J. Sofield 


\title{
Energy systematics of single and double $K$-shell-vacancy production in titanium bombarded by chlorine ions
}

\author{
James Hall and Patrick Richard \\ J. R. Macdonald Laboratory, Kansas State University, Manhattan, Kansas 66506
}

Philip L. Pepmiller, D. C. Gregory, P. D. Miller, C. D. Moak, C. M. Jones, and G. D. Alton Physics Division, Oak Ridge National Laboratory, Oak Ridge, Tennessee 37831

L. B. Bridwell

Physics Department, Southwest Missouri State University, Springfield, Missouri 65806

C. J. Sofield

Nuclear Physics Division, U.K.A.E.A., Atomic Energy Research Establishment, Harwell, Didcot, Oxon OX11 ORA, United Kingdom

(Received 5 August 1985)

\begin{abstract}
The energy systematics of single and double $K$-shell-vacancy production in titanium have been investigated near the limit of zero target thickness for incident chlorine ions over an energy range of 7-15 MeV/amu. Single and double $K$-shell-vacancy production cross sections have been measured for projectiles incident with zero, one, and two initial $K$-shell vacancies. Single and double $K$-shellto- $K$-shell electron-transfer cross sections have been obtained indirectly using differencing procedures based on the projectile-charge-state dependence of single and double $K$-shell-vacancy production. The measured cross sections have been compared to theoretical models for direct ionization and inner-shell electron transfer. Together with previously reported data, these cross sections also have been used to investigate the relative importance of these mechanisms for $K$-shell-vacancy production in heavy-ion-atom collisions over a range of scaled velocities of $0.24 \leq V_{1} / V_{2 K} \leq 1.29$.
\end{abstract}

\section{INTRODUCTION}

Direct ionization and electron transfer are two of the principal mechanisms responsible for inner-shell-vacancy production in ion-atom collisions. ${ }^{1}$ In recent work Hall et $a .^{2-5}$ have investigated the relative importance of these mechanisms in heavy-ion-atom collisions by measuring the projectile energy, atomic number, and charge-state dependence of $K$-shell-vacancy production near the limit of zero target thickness $\left(\sim 1 \mu \mathrm{g} / \mathrm{cm}^{2}\right)$ for titanium bombarded by $\mathrm{C}, \mathrm{N}, \mathrm{O}, \mathrm{F}, \mathrm{Mg}, \mathrm{Al}, \mathrm{Si}, \mathrm{S}$, and $\mathrm{Cl}$ ions $\left(0.27 \leq Z_{1} / Z_{2} \leq 0.77\right)$ over a maximum energy range of 0.5 to $6.5 \mathrm{MeV} / \mathrm{amu}\left(0.24 \leq V_{1} / V_{2 K} \leq 0.85\right.$, where $V_{1}$ is the projectile nuclear velocity and $V_{2 K}$ is the mean velocity of an electron in the target $K$ shell). Direct ionization was found to play a dominant role in both single- and double-target $K$-shell-vacancy production by multielectron ions $\left(q \leq Z_{1}-3\right)$ in asymmetric collision systems $\left(Z_{1} / Z_{2} \leq 0.40\right)$ at low to intermediate collision velocities $\left(V_{1} / V_{2 K} \leq 0.8\right)$. As $Z_{1} / Z_{2}$ increases toward unity (symmetric systems), electron transfer from the target $K$ shell to outer shells of the projectile was found to play an increasingly important role in $K$-shell-vacancy production at low scaled velocities $\left(V_{1} / V_{2 K} \leq 0.5\right)$. For the case of bare incident ions $\left(q=Z_{1}\right)$, single and/or double $K$-shellto- $K$-shell electron transfer was found to make a significant contribution to target $K$-shell-vacancy production in asymmetric systems as low as $Z_{1} / Z_{2} \sim 0.25$ and to dominate target $K$-shell-vacancy production in near- symmetric collision systems $\left(Z_{1} / Z_{2} \geq 0.75\right)$ at low scaled velocities $\left(V_{1} / V_{2 K} \leq 0.5\right)$. The present work extends the range of previously reported cross sections ${ }^{2-5}$ for the case of $\mathrm{Cl}+\mathrm{Ti}\left(Z_{1} / Z_{2}=0.77\right)$ well beyond the region of matched collision velocity for the target $K$ shell $\left(V_{1} / V_{2 K} \sim 1.0\right)$, where many of the vacancy-production mechanisms are expected to maximize. Together with the previous data, the present work provides a broad range of self-consistent data for direct ionization and inner-shell electron transfer spanning the range $0.24 \leq V_{1} / V_{2 K}$ $\leq 1.29$. The measured cross sections are compared with perturbative one-and-a-half-center ${ }^{6-10}$ (POHCE) and modified perturbed-stationary-state ${ }^{11-14}$ (ECPSSR) calculations for direct ionization plus electron transfer and with nonperturbative two-center, two-state atomicexpansion $^{15-18}$ (TSAE) calculations for $K$-shell-to- $K$-shell electron transfer.

\section{EXPERIMENTAL PROCEDURE}

The present experiment was performed at the Holifield Heavy-Ion Research Facility (HHIRF) at Oak Ridge National Laboratory. Chlorine beams in the 7-15 $\mathrm{MeV} / \mathrm{amu}$ range were obtained from the Oak Ridge Isochronous Cyclotron (ORIC) operated as a high-energy booster to a National Electrostatics Corporation 25-MV folded tandem van de Graaff accelerator. The ion beams, obtained in well-defined charge states corresponding to zero, one, and two initial $K$-shell vacancies, were then 
focused onto thin $\left(\sim 1 \mu \mathrm{g} / \mathrm{cm}^{2}\right)$ transmission-mounted Ti-foil targets oriented at $45^{\circ}$ with respect to the beam axis. Extremely thin targets were used in order to approach single-collision conditions and thus avoid ambiguities in $\mathrm{x}$-ray-yield measurements due to target-thickness effects. ${ }^{19}$ The yield of $\mathrm{Ti} K \times$ rays per unit of integrated beam current was determined for each projectile energy and incident charge state using a solid-state $\mathrm{Si}(\mathrm{Li})$ detector (resolution $\sim 150 \mathrm{eV}$ at $5.9 \mathrm{keV}$ ) mounted in vacuum at a laboratory angle of $90^{\circ}$ with respect to the beam axis [cf. Fig. 1(a)]. The charge-state distribution of the beam leaving the target foil was determined by electrostatic analysis in a separate measurement using a position-sensitive detector [cf. Fig. 1(b)]. Effective target $K$ x-ray production cross sections were then calculated from

$$
\sigma_{K \mathrm{x}}(\text { tot })=\frac{Y_{K \mathrm{x}}(\text { tot })}{n_{p} \rho x \xi d \Omega},
$$

where $\xi d \Omega\left(\sim 8 \times 10^{-5}\right)$ represents the counting efficiency of the $\mathrm{Si}(\mathrm{Li})$ detector (measured in situ using a calibrated ${ }^{55} \mathrm{Fe}$ source), $\rho x\left(\sim 1 \times 10^{16}\right.$ atoms $\left./ \mathrm{cm}^{2}\right)$ represents the target areal density (measured using proton backscattering), and $n_{p}$ represents the total number of incident particles during a given run (determined using integrated beam currents and the measured charge-state distributions).

The energy resolution of the detector was sufficient to distinguish the hypersatellite $\mathrm{x}$ rays due to the decay of double- $K$-vacancy states from the satellite $\mathrm{x}$ rays due to the decay of single- $K$-vacancy states. A least-squares analysis of the Ti $\mathrm{x}$-ray spectra thus allowed the total $\mathrm{x}$ ray production cross section $\sigma_{K x}$ (tot) to be separated into satellite and hypersatellite components as shown in Fig. 1(a). The cross section for hypersatellite production, $\sigma_{K \mathrm{x}}(H)$, was obtained from a ratio of $\mathrm{x}$-ray yields according to

$$
\sigma_{K \mathrm{x}}(H)=\sigma_{K \mathrm{x}}(\text { tot })\left[Y_{K \alpha} h / Y_{K \alpha}(\text { tot })\right]=\bar{\omega}_{K} \sigma_{\mathrm{DKV}},
$$

where $\sigma_{\mathrm{DKV}}$ represents the double- $K$-vacancy production cross section. The satellite component, corrected for cascade from double- $K$-vacancy states, was obtained from

$$
\sigma_{K \mathrm{x}}(S)=\sigma_{K \mathrm{x}}(\text { tot })-2 \sigma_{K \mathrm{x}}(H)=\bar{\omega}_{K} \sigma_{\mathrm{SKV}},
$$

where $\sigma_{\mathrm{SKV}}$ represents the single- $K$-vacancy production cross section and where we have assumed that the hypersatellite fluorescence yield is equal to the average $K$-shell fluorescence yield $\bar{\omega}_{K} \cdot 2,5$

The relative uncertainties in experimental values of $\sigma_{K \mathrm{x}}(S)$ and $\sigma_{K \mathrm{x}}(H)$ are $15-20 \%$ and $20-25 \%$, respectively. The absolute uncertainties in experimental values of $\sigma_{K \mathrm{x}}(S)$ and $\sigma_{K \mathrm{x}}(H)$ are estimated to be $5-10 \%$ greater than the relative uncertainties in each case. Experimental values for $\sigma_{K \mathrm{x}}($ tot $), \sigma_{K \mathbf{x}}(S)$, and $\sigma_{K \mathrm{x}}(H)$ are given in Table I.

Single and double $K$-shell-to- $K$-shell electron-transfer cross sections were obtained indirectly using differencing procedures based on the projectile charge-state dependence of single- and double- $K$-vacancy production. Under the assumption that the average $K$-shell fluorescence yield and the contributions to single- $K$-vacancy production due to processes other than $K$-shell-to- $K$-shell transfer are in-
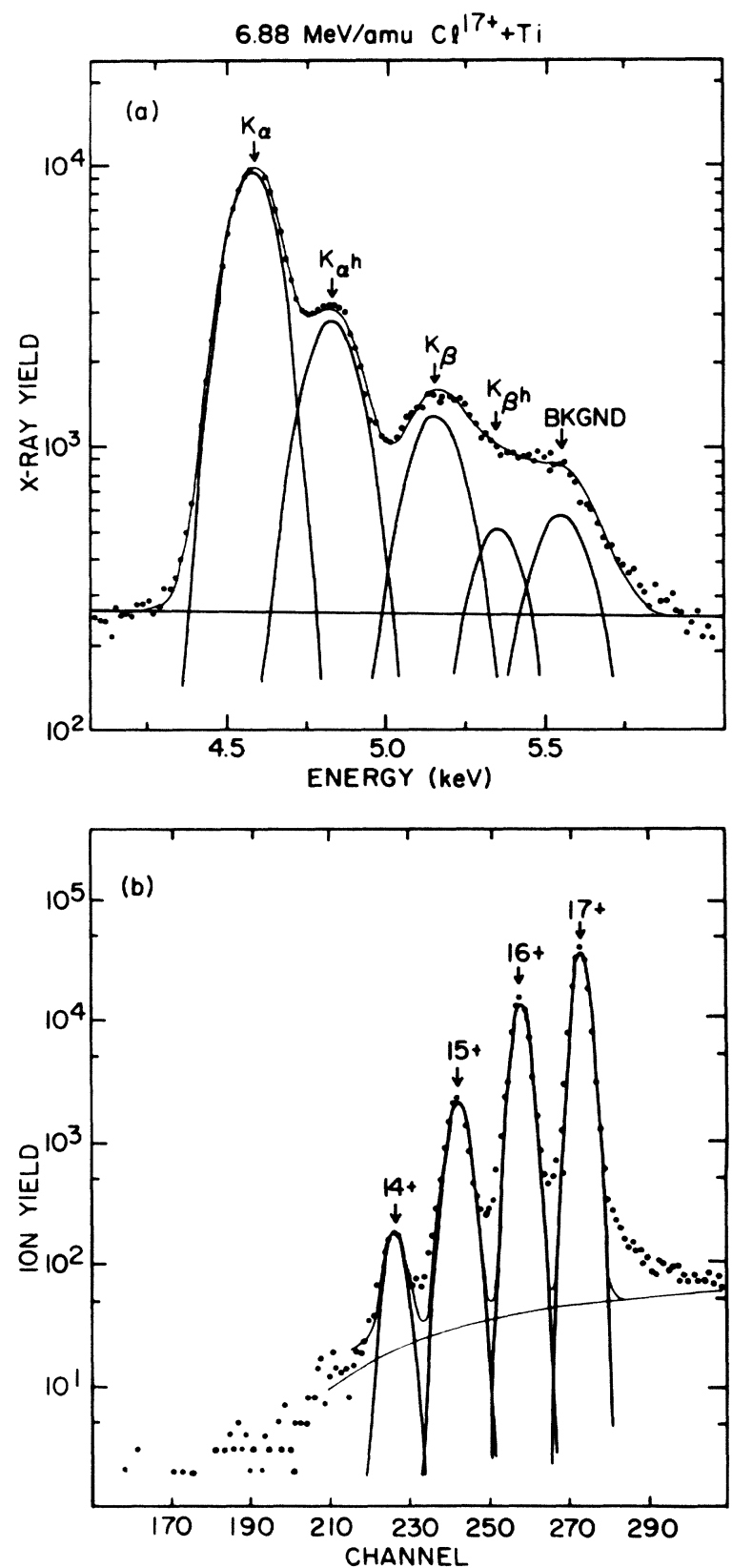

FIG. 1. (a) Titanium $K \mathrm{x}$-ray spectrum observed in low resolution for $6.88 \mathrm{MeV} / \mathrm{amu} \mathrm{Cl}{ }^{17+}+\mathrm{Ti}$. The principal features of the $x$-ray-energy spectrum are shown beneath the data. The high-energy feature labeled BKGND is an impurity peak associated with the target frame. (b) Charge-state distribution of the chlorine beam leaving the target foil for $6.88 \mathrm{MeV} / \mathrm{amu}$ $\mathrm{Cl}^{17+}+\mathrm{Ti}$. The principal charge states, as stripped out by least-squares analysis, are shown beneath the data.

dependent of the incident charge state, the single $K$-shellto- $K$-shell electron-transfer cross section may be expressed in terms of the $\sigma_{\mathrm{SKV}}^{i}$ (where the superscript $i=0,1,2$ refers to the number of initial $K$-shell vacancies) as

$$
\sigma_{\text {SKV }}^{K-K}=\sigma_{\text {SKV }}^{2}-\sigma_{\text {SKv }}^{0} .
$$

Under similar assumptions, the double $K$-shell-to- $K$-shell electron-transfer cross section may be expressed as

$$
\sigma_{\mathrm{DKV}}^{2 K-2 K}=\sigma_{\mathrm{DKV}}^{2}-2 \sigma_{\mathrm{DKV}}^{1}+\sigma_{\mathrm{DKV}}^{0} .
$$


TABLE I. $K$-shell x-ray production cross sections for $\mathrm{Cl}^{q+}+\mathrm{Ti}\left(\mathrm{cm}^{2} /\right.$ atom). Numbers in parentheses indicate powers of 10 .

\begin{tabular}{|c|c|c|c|c|c|}
\hline$E(\mathrm{MeV} / \mathrm{amu})^{\mathrm{a}}$ & $\bar{\omega}_{K}^{\mathrm{b}}$ & $q+$ & $\sigma_{K \mathrm{x}}($ tot $)$ & $\sigma_{K \mathbf{x}}(S)$ & $\sigma_{K \mathbf{x}}(H)$ \\
\hline 0.50 & 0.239 & $5+$ & $1.12 \pm 0.24(-21)$ & $1.12 \pm 0.24(-21)$ & \\
\hline 0.75 & 0.245 & $6+$ & $3.52 \pm 0.75(-21)$ & $3.52 \pm 0.75(-21)$ & \\
\hline 1.00 & 0.248 & $6+$ & $7.39 \pm 1.57(-21)$ & $7.39 \pm 1.57(-21)$ & \\
\hline 1.25 & 0.249 & $7+$ & $1.30 \pm 0.28(-20)$ & $1.30 \pm 0.28(-20)$ & \\
\hline 1.50 & 0.249 & $5+$ & $1.65 \pm 0.35(-20)$ & $1.65 \pm 0.35(-20)$ & \\
\hline 1.75 & 0.248 & $6+$ & $2.35 \pm 0.50(-20)$ & $2.35 \pm 0.50(-20)$ & \\
\hline 2.00 & 0.247 & $6+$ & $2.82 \pm 0.60(-20)$ & $2.75 \pm 0.61(-20)$ & $3.38 \pm 0.88(-22)$ \\
\hline 3.00 & 0.243 & $8+$ & $5.40 \pm 1.15(-20)$ & $5.07 \pm 1.11(-20)$ & $1.67 \pm 0.43(-21)$ \\
\hline 4.00 & 0.238 & $10+$ & $7.76 \pm 1.65(-20)$ & $7.36 \pm 1.62(-20)$ & $2.02 \pm 0.52(-21)$ \\
\hline 5.00 & 0.232 & $14+$ & $1.17 \pm 0.25(-19)$ & $1.06 \pm 0.23(-19)$ & $5.50 \pm 1.43(-21)$ \\
\hline 6.88 & 0.219 & $11+$ & $1.27 \pm 0.28(-19)$ & $1.15 \pm 0.25(-19)$ & $5.68 \pm 1.53(-21)$ \\
\hline 9.00 & 0.217 & $12+$ & $2.31 \pm 0.51(-19)$ & $2.07 \pm 0.46(-19)$ & $1.25 \pm 0.34(-20)$ \\
\hline 10.67 & 0.216 & $12+$ & $3.56 \pm 0.78(-19)$ & $3.20 \pm 0.70(-19)$ & $1.85 \pm 0.50(-20)$ \\
\hline 12.62 & 0.216 & $13+$ & $2.55 \pm 0.56(-19)$ & $2.09 \pm 0.46(-19)$ & $2.27 \pm 0.60(-20)$ \\
\hline 15.05 & 0.216 & $14+$ & $4.06 \pm 0.89(-19)$ & $3.33 \pm 0.73(-19)$ & $3.65 \pm 0.99(-20)$ \\
\hline 0.50 & 0.239 & $16+$ & & & \\
\hline 0.75 & 0.245 & $16+$ & & & \\
\hline 1.00 & 0.248 & $16+$ & & & \\
\hline 1.25 & 0.249 & $16+$ & & & \\
\hline 1.50 & 0.249 & $16+$ & & & \\
\hline 1.75 & 0.248 & $16+$ & $2.86 \pm 0.61(-19)$ & $2.86 \pm 0.61(-19)$ & \\
\hline 2.00 & 0.247 & $16+$ & $3.50 \pm 0.74(-19)$ & $3.36 \pm 0.74(-19)$ & $7.00 \pm 1.82(-21)$ \\
\hline 3.00 & 0.243 & $16+$ & $3.90 \pm 0.83(-19)$ & $3.71 \pm 0.82(-19)$ & $9.75 \pm 2.53(-21)$ \\
\hline 4.00 & 0.238 & $16+$ & $5.44 \pm 1.15(-19)$ & $4.86 \pm 1.07(-19)$ & $2.88 \pm 0.75(-20)$ \\
\hline 5.00 & 0.232 & $16+$ & $5.28 \pm 1.12(-19)$ & $4.53 \pm 1.00(-19)$ & $3.75 \pm 0.97(-20)$ \\
\hline 6.88 & 0.219 & $16+$ & $4.15 \pm 0.91(-19)$ & $3.37 \pm 0.73(-19)$ & $3.80 \pm 1.04(-20)$ \\
\hline 9.00 & 0.217 & $16+$ & $4.80 \pm 1.05(-19)$ & $3.82 \pm 0.84(-19)$ & $4.84 \pm 1.30(-20)$ \\
\hline 10.67 & 0.216 & $16+$ & $4.86 \pm 1.07(-19)$ & $3.84 \pm 0.84(-19)$ & $4.99 \pm 1.34(-20)$ \\
\hline 12.62 & 0.216 & $16+$ & $3.89 \pm 0.86(-19)$ & $2.89 \pm 0.63(-19)$ & $4.97 \pm 1.34(-20)$ \\
\hline 15.05 & 0.216 & $16+$ & $5.14 \pm 1.13(-19)$ & $4.00 \pm 0.89(-19)$ & $5.66 \pm 1.53(-20)$ \\
\hline 0.50 & 0.239 & $17+$ & & & \\
\hline 0.75 & 0.245 & $17+$ & & & \\
\hline 1.00 & 0.248 & $17+$ & & & \\
\hline 1.25 & 0.249 & $17+$ & & & \\
\hline 1.50 & 0.249 & $17+$ & & & \\
\hline 1.75 & 0.248 & $17+$ & & $3.14 \pm 0.79(-19)^{c}$ & \\
\hline 2.00 & 0.247 & $17+$ & & $3.68 \pm 0.92(-19)^{\mathrm{c}}$ & \\
\hline 3.00 & 0.243 & $17+$ & $8.95 \pm 1.90(-19)$ & $5.19 \pm 1.14(-19)$ & $1.88 \pm 0.44(-19)$ \\
\hline 4.00 & 0.238 & $17+$ & $9.24 \pm 1.96(-19)$ & $4.80 \pm 1.06(-19)$ & $2.22 \pm 0.52(-19)$ \\
\hline 5.00 & 0.232 & $17+$ & $8.51 \pm 1.81(-19)$ & $4.46 \pm 0.98(-19)$ & $2.03 \pm 0.48(-19)$ \\
\hline 6.88 & 0.219 & $17+$ & $9.09 \pm 2.00(-19)$ & $4.80 \pm 1.06(-19)$ & $2.14 \pm 0.58(-19)$ \\
\hline 9.00 & 0.217 & $17+$ & $1.04 \pm 0.23(-18)$ & $5.90 \pm 1.30(-19)$ & $2.22 \pm 0.60(-19)$ \\
\hline 10.67 & 0.216 & $17+$ & $9.66 \pm 2.12(-19)$ & $6.03 \pm 1.32(-19)$ & $1.82 \pm 0.49(-19)$ \\
\hline 12.62 & 0.216 & $17+$ & $6.26 \pm 1.38(-19)$ & $3.78 \pm 0.84(-19)$ & $1.23 \pm 0.33(-19)$ \\
\hline 15.05 & 0.216 & $17+$ & $6.67 \pm 1.47(-19)$ & $4.43 \pm 0.97(-19)$ & $1.13 \pm 0.30(-19)$ \\
\hline
\end{tabular}

${ }^{a}$ The range of measured cross sections tabulated here has been extended down to $0.50 \mathrm{MeV} / \mathrm{amu}$ by including previously reported data measured at Brookhaven National Laboratory (Refs. 2 and 5). These data are reprinted in the present work for completeness.

${ }^{b}$ Average $K$-shell fluorescence yields determined from the intensity distribution of the $K \alpha$ satellite peaks observed in high resolution (cf. Ref. 2)

${ }^{c}$ Cross sections estimated from existing data using the charge-state dependence of $\sigma_{K \mathrm{x}}(S)$ (cf. Ref. 2).

These procedures have been used in several previous publications. $^{2-5,19}$

The relative uncertainties in experimental values of $\sigma_{\mathrm{SKV}}^{K-K}$ and $\sigma_{\mathrm{DKV}}^{2 K-2 K}$ range from $30 \%$ at the lowest projectile energies measured to $50 \%$ and $90 \%$, respectively, at the highest energies, primarily due to the fact that Eqs. (4) and (5) require measuring relatively small differences be- tween large quantities in the high-velocity regime $\left(V_{1} / V_{2 K} \geq 1\right)$. The absolute uncertainties in $\sigma_{\text {SKV }}^{K-K}$ and $\sigma_{\mathrm{DKV}}^{2 R-2 K}$ are estimated to be $5-10 \%$ greater than the relative uncertainties in each case. Experimental values for $\sigma_{\mathrm{SKV}}^{K-K}$ and $\sigma_{\mathrm{DKV}}^{2 K-2 K}$ presented in the present work are derived from Eqs. (4) and (5), respectively, using the values of $\sigma_{K \mathbf{x}}(S)$ and $\sigma_{K \mathrm{x}}(H)$ given in Table I. 


\section{DISCUSSION}

The projectile energy dependence of single- $K$-vacancy production for the $\mathrm{Cl}^{q+}+\mathrm{Ti}$ system is shown in Fig. 2 . Experimental points are derived from satellite $x$-ray production cross sections for projectiles incident with zero ( $\left.\sigma_{\mathrm{SKV}}^{0}, q \leq 14\right)$ and two $\left(\sigma_{\mathrm{SKV}}^{2}, q=17\right.$ ) initial $K$-shell vacancies. Cross sections for two-electron projectile ions ( $q=15$ ) are not considered here due to the metastable $1 s 2 s{ }^{3} S$ component $(\tau \sim 0.4 \mu \mathrm{s})$ in the incident beam. The range of measured cross sections shown in Fig. 2 has been extended down to $0.50 \mathrm{MeV} / \mathrm{amu}\left(V_{1} / V_{2 K}=0.24\right)$ by including previously reported data measured at Brookhaven National Laboratory. ${ }^{2-5}$ Recently reported data due to Tanis et al. ${ }^{20}$ for multielectron ions ( $\left.\sigma_{\mathrm{SKV}}^{0}, q \leq 14\right)$ are also shown in Fig. 2. These data cover the velocity range $0.25 \leq V_{1} / V_{2 K} \leq 0.50$ and agree quite well with our previous work. Although generally reliable $a b$ initio calculations for inner-shell-vacancy production in heavy, nearsymmetric collision systems $\left(Z_{1} / Z_{2} \sim 1\right)$ at low to intermediate scaled velocities $\left(V_{1} / V_{2 K} \leq 1.0\right)$ are not yet available, two theoretical models are presented in Fig. 2 for

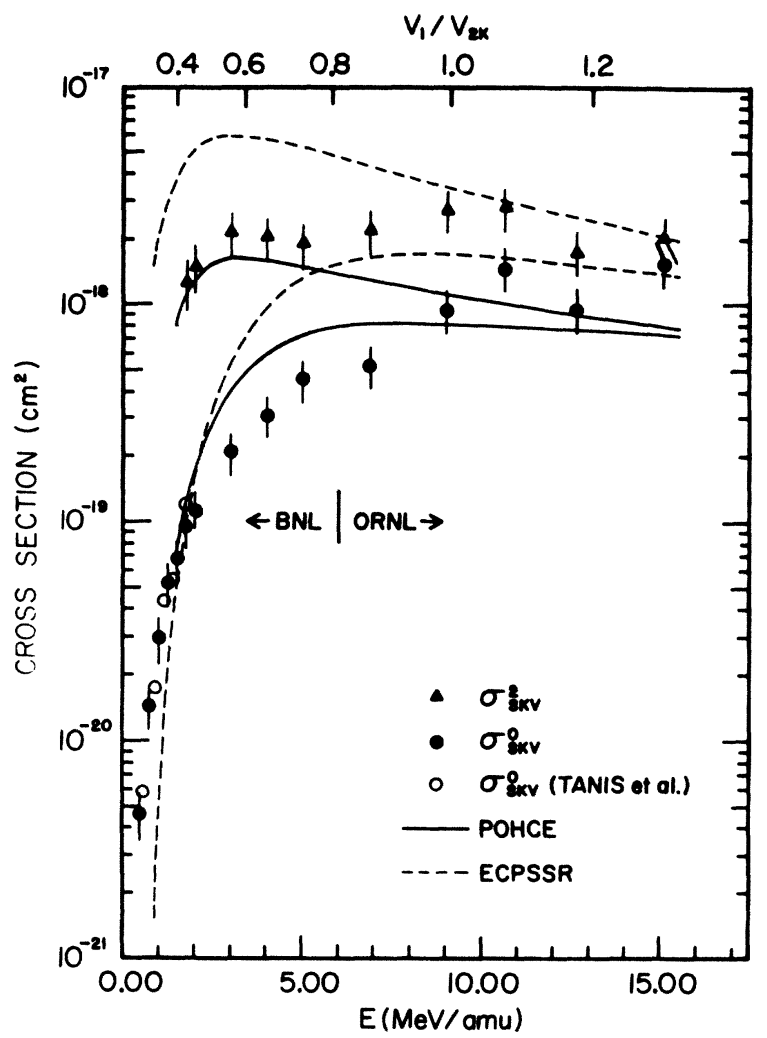

FIG. 2. Energy dependences of single- $K$-vacancy production for incident bare ( $\left.\sigma_{\mathrm{SKV}}^{2}, q=17\right)$ and multielectron $\left(\sigma_{\mathrm{SKV}}^{0}, q \leq 14\right)$ projectiles. Previously reported data due to Hall et al. (Refs. 2-5) and recently published data due to Tanis et al. (Ref. 20) are shown for $V_{1} / V_{2 K} \leq 0.8$. The data due to Tanis et al. have been plotted using the fluorescence yields suggested in Table $I$. The solid lines represent POHCE calculations (Refs. 6-10) for direct ionization plus $K \rightarrow L M N$... electron transfer (lower curve) and direct ionization plus $K \rightarrow K L M N \ldots$ electron transfer (upper curve). The dashed lines represent ECPSSR calculations (Refs. 11-14) for direct ionization plus $K \rightarrow L M N$. . electron transfer (lower curve) and direct ionization plus $K \rightarrow K L M N$. . . electron transfer (upper curve). comparison with data. The solid lines represent POHCE calculations for direct ionization plus electron transfer to outer shells of the projectile (lower curve) and direct ionization plus electron transfer to all shells of the projectile (upper curve) based on the coupled-channel approach of Becker et al. ${ }^{6-10}$ POHCE calculations describe the time-dependent wave function of the active electron in terms of a one-center, multistate atomic expansion about the target and account for small-amplitude electron transfer by including appropriate projectile-centered final states. The dashed lines represent ECPSSR calculations for direct ionization plus electron transfer to outer shells of the projectile (lower curve) and direct ionization plus electron transfer to all shells of the projectile (upper curve) based on the modified perturbed-stationary-state approach of Lapicki et al. ${ }^{11-14}$ ECPSSR calculations for direct ionization ${ }^{12}$ describe the target-electron wave functions under the influence of the projectile as perturbed stationary states and include projectile energy-loss $(E)$, Coulomb deflection $(C)$, and relativistic $(R)$ corrections. Electron transfer is accounted for in these calculations through the use of a semiempirical interpolation formu$\mathrm{la}^{13,14}$ linking modified Oppenheimer-Brinkman-Kramers (OBK) calculations at low velocities $\left(V_{1} / V_{2 K} \ll 1\right)$ with calculations obtained in the second Born approximation at high velocities $\left(V_{1} / V_{2 K} \gg 1\right)$.

Single- $K$-vacancy production by multielectron ions ( $\sigma_{\mathrm{SKV}}^{0}, q \leq 14$ ) shows a rapid rise with increasing velocity at low scaled velocities $\left(V_{1} / V_{2 K} \leq 0.50\right)$. ECPSSR calculations, which assume a fully vacant projectile $L$ shell, underestimate measured $\sigma_{\text {SKV }}^{0}$ cross sections by factors of $5-10$ at the lowest velocities measured here, but become comparable in magnitude to measured cross sections for $V_{1} / V_{2 K} \sim 0.50$. Similar deviations of ECPSSR theory from experiment have been observed by Tanis et al. ${ }^{20}$ for heavy, near-symmetric collision systems at low scaled velocities and are not at all unexpected since the theory is intended to apply only to collision systems for which $Z_{1} / Z_{2} \leq 0.5$. Over the range $0.50 \leq V_{1} / V_{2 K} \leq 1.0$, ECPSSR calculations tend to overestimate measured cross sections by factors of 2-3. ECPSSR calculations predict a continued steep rise with increasing velocity toward a broad maximum in the cross section near matched velocity $\left(V_{1} / V_{2 K} \sim 1.0\right)$, while the measured cross sections show a more gradual rise with increasing velocity and show no evidence of a maximum in this velocity range. At higher velocities, where single- $K$-vacancy production by multielectron ions is expected to be dominated by direct ionization, ECPSSR calculations are in reasonably good agreement with the magnitude of the measured cross sections. POHCE calculations (available only for $V_{1} / V_{2 K} \geq 0.40$ ) are comparable in magnitude to our data at low scaled velocities $\left(V_{1} / V_{2 K} \sim 0.40\right)$, but tend to overestimate measured $\sigma_{\mathrm{SKV}}^{0}$ cross sections by up to a factor of 2 over the range $0.50 \leq V_{1} / V_{2 K} \leq 1.0$. At higher velocities, POHCE calculations tend to underestimate measured cross sections by roughly a factor of 2 . Although the data do not extend far enough to test the energy dependence of these calculations at high velocities, it is apparent that the maximum in the cross section will occur at a significantly higher scaled velocity than predicted by 
these theories.

Single- $K$-vacancy production by bare ions $\left(\sigma_{\mathrm{SKV}}^{2}\right.$, $q=17)$ at low to intermediate scaled velocities $\left(V_{1} / V_{2 K} \leq 1.0\right)$ is observed to exceed that for multielectron ions by factors of $2-10$ for the case of $\mathrm{Cl}^{17+}+\mathrm{Ti}$. The difference between the two cross sections is attributed to the addition of the $K$-shell-to- $K$-shell electron-transfer channel. At higher velocities, where the relative importance of $K$-to- $K$ electron transfer is expected to be diminished, the cross sections for single- $K$-vacancy production by bare and multielectron ions become comparable in magnitude. The measured cross sections for single- $K$ vacancy production by bare ions exhibit a rise to a broad plateau extending from $V_{1} / V_{2 K} \sim 0.50$ up to the limit of the present data. ECPSSR calculations overestimate measured $\sigma_{\mathrm{SKV}}^{2}$ cross sections at low to intermediate scaled velocities $\left(V_{1} / V_{2 K} \leq 1.0\right)$ by factors of $2-3$, but are in reasonably good agreement with the magnitude of the measured cross sections at high velocities. This result is consistent with the earlier work of Lapicki et al., ${ }^{13,14}$ which indicates that the electron-transfer theory of Refs. 13 and 14 tends to lie above experiment in heavy, nearsymmetric collision systems $\left(Z_{1} / Z_{2} \sim 1\right)$. POHCE calculations are in reasonably good agreement with the magnitude of the measured $\sigma_{\mathrm{SKV}}^{2}$ cross sections at low scaled velocities $\left(V_{1} / V_{2 K} \leq 0.7\right)$, but tend to underestimate measured cross sections by factors of $2-3$ at higher velocities.

The projectile energy dependence of double- $K$-vacancy production for the $\mathrm{Cl}^{q+}+\mathrm{Ti}$ system is shown in Fig. 3 . Experimental points are derived from hypersatellite $\mathrm{x}$-ray production cross sections for projectiles incident with zero $\left(\sigma_{\mathrm{DKV}}^{0}, q \leq 14\right)$, one $\left(\sigma_{\mathrm{DKV}}^{1}, q=16\right)$, and two $\left(\sigma_{\mathrm{DKV}}^{2}\right.$, $q=17$ ) initial $K$-shell vacancies. The range of measured cross sections shown in Fig. 3 has been extended down to $2.0 \mathrm{MeV} / \mathrm{amu}\left(V_{1} / V_{2 K} \sim 0.5\right)$ by including previously reported data measured at Brookhaven National Laboratory. $^{2-5}$ The solid lines represent POHCE calculations for double- $K$-shell-vacancy production.

Double- $K$-vacancy production by multielectron ions ( $\sigma_{\mathrm{DKV}}^{0}, q \leq 14$ ) falls $1-2$ orders of magnitude below that for single- $K$-vacancy production, although the two processes show generally similar systematics. POHCE calculations for double- $K$-vacancy production by multielectron ions overestimate measured $\sigma_{\mathrm{DKV}}^{0}$ cross sections by factors of 2-5 at low to intermediate scaled velocities $\left(V_{1} / V_{2 K} \leq 1.1\right)$. The calculations predict a steep rise with increasing velocity toward a maximum in the cross section near matched velocity $\left(V_{1} / V_{2 K} \sim 1.0\right)$, while the measured cross sections show a more gradual rise with increasing velocity and, as was the case for single- $K$ vacancy production, show no evidence of a maximum in this velocity range.

Double- $K$-vacancy production by bare ions $\left(\sigma_{\mathrm{DKV}}^{2}\right.$, $q=17$ ) falls a factor of $2-4$ below that for single- $K$ vacancy production, although, again, the two processes show generally similar systematics. Double- $K$-vacancy production by bare ions is observed to exceed that for multielectron ions by 1-2 orders of magnitude for the case of $\mathrm{Cl}^{17+}+\mathrm{Ti}$ at low to intermediate scaled velocities $\left(V_{1} / V_{2 K} \leq 1.0\right)$. The difference between the two cross sections is attributed to the addition of two processes: (1)

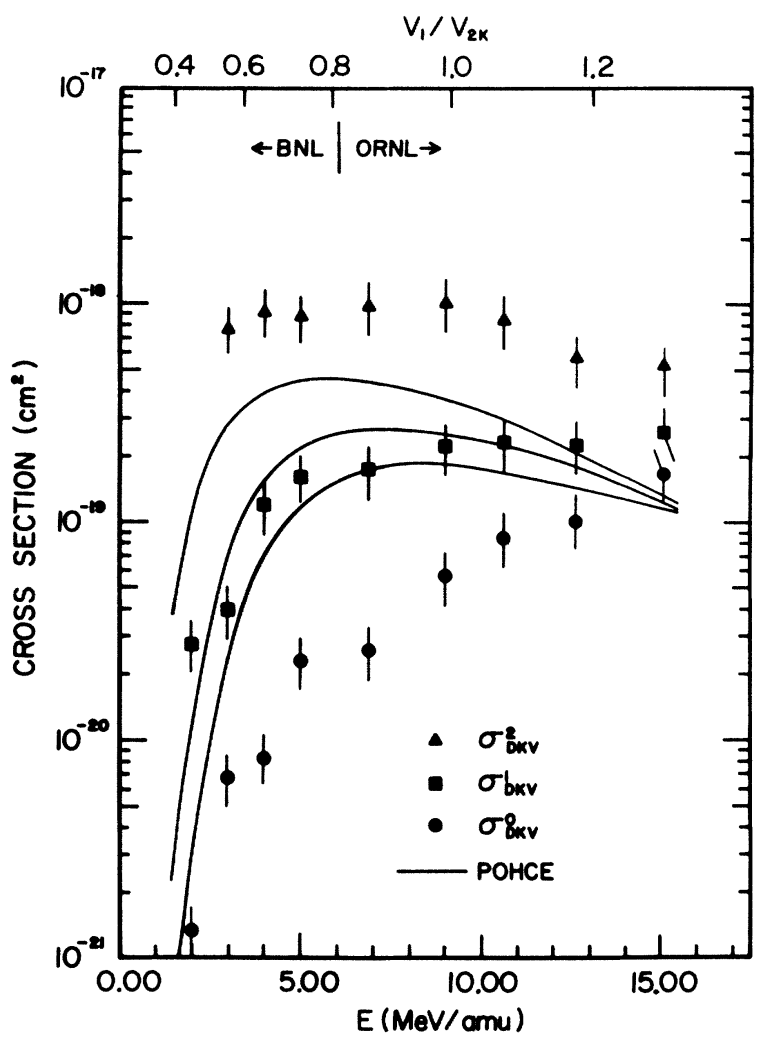

FIG. 3. Energy dependences of double- $K$-vacancy production for incident bare $\left(\sigma_{\mathrm{DKV}}^{2}, q=17\right)$, one-electron ( $\left.\sigma_{\mathrm{DKV}}^{1}, q=16\right)$, and multielectron $\left(\sigma_{\mathrm{DKV}}^{0}, q \leq 14\right)$ projectiles. Previously reported data due to Hall et al. (Refs. 2-5) are shown for $V_{1} / V_{2 K} \leq 0.8$. The solid lines represent POHCE calculations (Refs. 6-10) for direct ionization plus appropriate electrontransfer contributions.

direct ionization or electron transfer to outer shells of the projectile simultaneous with single $K$-shell-to- $K$-shell electron transfer, and (2) double $K$-to- $K$ electron transfer. At higher velocities, where the relative importance of $K$-to- $K$ electron transfer is expected to be diminished, the cross sections for double- $K$-vacancy production by bare and multielectron ions differ by only a factor of $2-3$. POHCE calculations for double- $K$-vacancy production by bare ions underestimate measured $\sigma_{\mathrm{DKV}}^{2}$ cross sections by factors of 2-3 over the full range of scaled velocities considered in the present work, but do show reasonably good agreement with the observed energy dependence at low to intermediate scaled velocities $\left(V_{1} / V_{2 K} \leq 1.2\right)$.

Double- $K$-vacancy production by one-electron ions ( $\left.\sigma_{\mathrm{DKV}}^{1}, q=16\right)$ falls intermediate between that for bare and multielectron ions. The measured cross sections rise to a broad plateau extending from $V_{1} / V_{2 K} \sim 0.8$ up to the limit of the present data and are observed to exceed the corresponding cross sections for multielectron ions by factors of 2-10. The enhancement of the double- $K$-vacancy production cross section for incident one-electron ions over that for multielectron ions is attributed to the addition of direct ionization or electron transfer to outer shells of the projectile simultaneous with single $K$-shell-to- $K$ shell electron transfer. POHCE calculations for double$K$-vacancy production by one-electron ions generally fall 
within a factor of 2 of our measured cross sections, but fail to reproduce the observed energy dependence.

The projectile energy dependences of single and double $K$-shell-to- $K$-shell electron transfer for the $\mathrm{Cl}^{17+}+\mathrm{Ti}$ system are shown in Fig. 4. Experimental points are derived from satellite and hypersatellite $x$-ray production cross sections according to Eqs. (4) and (5), respectively. The theoretical curves represent the TSAE calculations of Lin et al. ${ }^{15-18}$ which allow one to obtain both single $(-\cdot-\cdot-)$ and double $(-\cdots-\cdot-) \quad K$-shell-to- $K$-shell electron-transfer cross sections from a single calculation, as long as electron-electron correlation effects are neglected.

Single $K$-shell-to- $K$-shell electron-transfer cross sections rise to a broad maximum extending from $V_{1} / V_{2 K} \sim 0.5$ to $V_{1} / V_{2 K} \sim 0.9$ and appear to fall off gradually at higher velocities. The measured cross sections for single $K$-to- $K$ electron transfer are observed to exceed the sum of all other single- $K$-vacancy production mechanisms by factors of $2-10$ for the case of $\mathrm{Cl}^{17+}+\mathrm{Ti}$ at low to intermediate scaled velocities $\left(V_{1} / V_{2 K} \leq 1.0\right)$ (cf. Fig. 2). TSAE calculations are in reasonable agreement

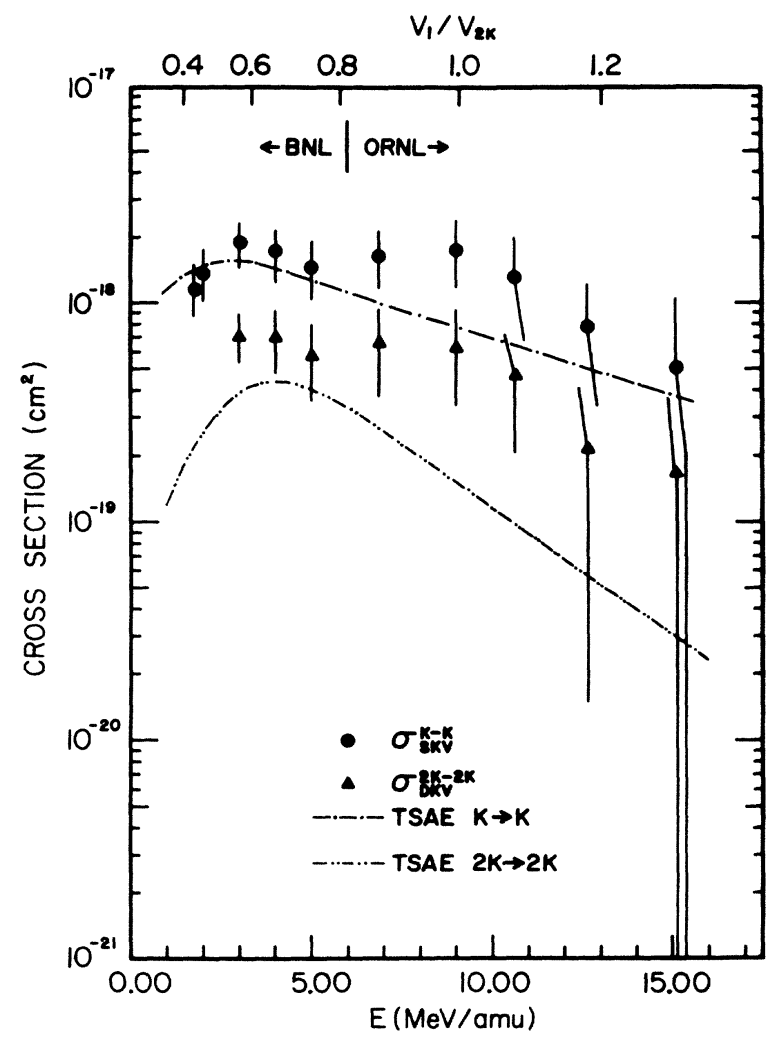

FIG. 4. Energy dependences of single $\left(\sigma_{\mathrm{SK} V}^{K-K}\right)$ and double $\left(\sigma_{\mathrm{DKV}}^{2 K-2 K}\right) K$-shell-to- $K$-shell electron transfer. Previously reported data due to Hall et al. (Refs. 2-5) are shown for $V_{1} / V_{2 K} \leq 0.8$. Statistical error bars are calculated based on the estimated uncertainties in $\sigma_{K \mathrm{x}}(S)$ and $\sigma_{K \mathrm{x}}(H)$ quoted in Table I. The theoretical curves represent TSAE $K \rightarrow K(-\cdot-\cdot)$ ) and $2 K \rightarrow 2 K \quad(-\cdots-\cdots-)$ electron-transfer calculations (Refs. 15-18). with experiment below $V_{1} / V_{2 K} \sim 0.8$, but appear to underestimate measured single $K$-to- $K$ electron-transfer cross sections at higher velocities. It should be noted, however, that above matched velocity $\left(V_{1} / V_{2 K} \sim 1.0\right)$ the cross sections for single- $K$-vacancy production by bare $\left(\sigma_{\mathrm{SKV}}^{2}, q=17\right)$ and multielectron $\left(\sigma_{\mathrm{SKV}}^{0}, q \leq 14\right)$ ions become comparable in magnitude and the differencing procedure used to obtain the single $K$-to- $K$ electron-transfer cross sections [Eq. (4)] becomes subject to large uncertainties.

Double $K$-shell-to- $K$-shell electron-transfer cross sections fall a factor of $2-3$ below those observed for single $K$-to- $K$ transfer, although the two processes show generally similar systematics. The measured cross sections for double $K$-to- $K$ electron transfer are observed to exceed the sum of all other double- $K$-vacancy production mechanisms by $1-2$ orders of magnitude for the case of $\mathrm{Cl}^{17+}+\mathrm{Ti}$ at low to intermediate scaled velocities $\left(V_{1} / V_{2 K} \leq 1.0\right.$ ) (cf. Fig. 3). TSAE calculations appear to underestimate measured double $K$-to- $K$ electron-transfer cross sections by factors of $2-5$ over the range of the present data, with the discrepancies becoming larger toward higher velocities. Once again, however, it should be noted that above matched velocity $\left(V_{1} / V_{2 K} \sim 1.0\right)$ the cross sections for double- $K$-vacancy production by bare $\left(\sigma_{\mathrm{PKV}}^{2}, q=17\right)$, one $\left(\sigma_{\mathrm{DKV}}^{1}, q=16\right)$, and multielectron ( $\sigma_{\mathrm{DKV}}, q<14$ ) ions become comparable in magnitude and the differencing procedure used to obtain the double $K$ to- $K$ electron-transfer cross sections [Eq. (5)] becomes subject to large uncertainties.

\section{SUMMARY}

In summary, the present work has extended the range of measured single- and double- $K$-shell-vacancy production cross sections for the $\mathrm{Cl}+\mathrm{Ti}$ collision system through the region of matched velocity for the target $K$ shell $\left(V_{1} / V_{2 K} \sim 1.0\right)$. Single- and double- $K$-vacancy production cross sections for bare ions $(q=17)$ showed generally similar energy systematics, each rising to a broad plateau at low to intermediate scaled velocities $\left(0.6 \leq V_{1} / V_{2 K} \leq 1.1\right)$ followed by a gradual falloff at higher velocities. Single- and double- $K$-vacancy production cross sections for multielectron ions $(q \leq 14)$ also showed generally similar systematics, each rising gradually toward a maximum beyond the range of the present data. The difference between the cross sections for $K$ vacancy production by bare and multielectron ions was attributed to the addition of processes involving single and/or double $K$-shell-to- $K$-shell electron transfer. Single and double $K$-to- $K$ electron transfer were observed to make a significant contribution to total $K$-vacancy production at low to intermediate scaled velocities $\left(V_{1} / V_{2 K} \leq 1.0\right)$ with measured cross sections exceeding the sum of all other $K$-vacancy production mechanisms by an order of magnitude or more for the case of $\mathrm{Cl}^{17+}+\mathrm{Ti}$. At higher velocities, where the relative importance of $K$-to- $K$ electron transfer is diminished, the cross sections for $K$-vacancy production by bare and multielectron ions were observed to become comparable in magnitude. 


\section{ACKNOWLEDGMENTS}

The authors would like to express their appreciation to R. L. Becker, G. Lapicki, and C. D. Lin for providing some of the calculations presented in this work prior to

${ }^{1}$ For a review of the study of direct ionization and electron transfer in ion-atom collisions, see C. D. Lin and P. Richard, Advances in Atomic and Molecular Physics, edited by D. Bates and B. Bederson (Academic, New York, 1981), Vol. 17, p. 275.

2J. Hall, Ph.D. dissertation, Kansas State University, 1981 (unpublished).

3J. Hall, P. Richard, T. J. Gray, C. D. Lin, K. Jones, B. Johnson, and D. Gregory, Phys. Rev. A 24, 2416 (1981).

4J. Hall, P. Richard, T. J. Gray, K. Jones, B. Johnson, and D. Gregory, Phys. Lett. 90A, 129 (1982).

5J. Hall, P. Richard, T. J. Gray, J. Newcomb, P. Pepmiller, C. D. Lin, K. Jones, B. Johnson, D. Gregory, Phys. Rev. A 28, 99 (1983); see AIP document No. PAPS PLRAA-28-99-32 for 32 pages of experimental cross sections (may be ordered from the American Institute of Physics, Physics Auxiliary Publication Service, 335 East 45th Street, New York, NY 10017).

${ }^{6}$ R. L. Becker (private communication).

7J. F. Reading and A. L. Ford, J. Phys. B 12, 1367 (1979).

${ }^{8}$ A. L. Ford, J. F. Reading, and R. L. Becker, J. Phys. B 12, publication. One of the authors (C.J.S.) would like to acknowledge travel support by a NATO grant which enabled him to participate in the experiment discussed in the present work. This work was supported by the Department of Energy, Division of Chemical Sciences, under Contract No. DE-AC02-76ER02753 and the Department of Energy, Division of Chemical Sciences and Office of Fusion Energy, under Contract No. DE-AC0584OR21400 with Martin Marietta Energy Systems, Inc.

2905 (1979).

${ }^{9}$ R. L. Becker, A. L. Ford, and J. F. Reading, J. Phys. B 13, 4059 (1980).

${ }^{10}$ A. L. Ford, J. F. Reading, and R. L. Becker, J. Phys. B 15, 3257 (1982).

${ }^{11} \mathrm{G}$. Lapicki (private communication).

12W. Brandt and G. Lapicki, Phys. Rev. A 23, 1717 (1981).

${ }^{13}$ G. Lapicki and W. Losonsky, Phys. Rev. A 15, 896 (1977).

${ }^{14}$ G. Lapicki and F. D. McDaniel, Phys. Rev. A 22, 1896 (1980).

${ }^{15}$ C. D. Lin (private communication).

${ }^{16}$ C. D. Lin, S. C. Soong, and L. N. Tunnell, Phys. Rev. A 17, 1646 (1978).

${ }^{17}$ C. D. Lin, Phys. Rev. A 19, 1510 (1979).

${ }^{18}$ C. D. Lin and L. N. Tunnell, Phys. Rev. A 22, 76 (1980).

${ }^{19}$ For a review, see T. J. Gray, Methods of Experimental Physics, edited by P. Richard (Academic, New York, 1980), Vol. 17, p. 193.

${ }^{20}$ J. A. Tanis, S. M. Shafroth, W. W. Jacobs, T. McAbee, and G. Lapicki, Phys. Rev. A 31, 750 (1985). 